\title{
CLASSIFICATION OF MICROCALCIFICATION BASED ON WAVE ATOM TRANSFORM
}

\author{
${ }^{1}$ Rajesh, A. and ${ }^{2}$ Mohan Ellappan \\ ${ }^{1}$ Department of ECE, Shri JIT University, Jhunjhunu, Rajasthan, India \\ ${ }^{2}$ Department of ECE, Pallavan College of Engineering, Kanchipuram, Tamilnadu, India
}

Received 2014-01-11; Revised 2014-03-12; Accepted 2014-04-05

\begin{abstract}
Breast cancer is a serious problem for women in the world. It is the most common form of cancer diagnosed in woman, with one in nine women expected to be diagnosed with some form of cancer in their life-time. It is second only to lung cancer in cancer related deaths. Statistics shows that the case of breast cancer in the world is increasing every year. By analyzing a mammogram, pathologists could detect the presence of microcalcification in ones breast. However, microcalcification can be classified into benign and malignant. The later indicates the presence of cancer. Computer-Aided Diagnosis (CADx) designed to help phatologists determine the type of microcalcification in a mammogram. Usually, it's consisting of two steps, feature extraction and classification. In our methodology, we proposed the use of Wave Atom Transform (WAT) as feature extraction technique and Support Vector Machine (SVM) as classifier. Using this methodology, our experimental result achieved good classification accuracy. However, some of the previous researches have shown better results than ours.
\end{abstract}

Keywords: Microcalcification, Support Vector Machine, Wave Atom Transform

\section{INTRODUCTION}

Classification of benign and malignant microcalcifications in mammograms through Computer aided Diagnosis (CADx) is vital for the early diagnosis of the breast cancer. Zhang and Gao (2007) applied the Translation Invariant (TI) wavelet transform to microcalcifications classification. The microcalcifications appear in small clusters of few pixels with relatively high intensity compared with their neighboring pixels.

Afrasiabi (2011) presented a blueprint based on one of the most fundamental laws governing the known universe, namely the second law of thermodynamics and I present support for its central role in initiation of mitosis and relationship of the other sub cellular compartments and their organization. Faye et al. (2009) described a new method of feature extraction from Wavelet coefficients for classification of digital mammograms. A matrix is constructed by putting
Wavelet coefficients of each image of a building set as a row vector. The method is tested using a set of images provided by the Mammographic Image Analysis Society (MIAS) to classify between normal and abnormal and then between benign and malignant tissues.

Ballesteros et al. (2002) evaluates the performance of applying a Computer Aided Diagnosis (CAD) system for the detection and classification of breast cancer in digital mammograms. The aim of this system is to detect microcalcifications and for this purpose it processes digitally the image, focused on the enhancement using the wavelet transform and a later classification.

Hamdi et al. (2008) has presented a high accuracy computer-aided diagnosis scheme. The goal of the developed system is to classify benign and malignant microcalcifications on mammograms. It is mainly based on a combination of wavelet decomposition, feature extraction and classification methodology using Fisher's linear discriminant. Jasmine et al. (2013) a novel approach for classifying microcalcification in digital

Corresponding Author: Rajesh, A., Department of ECE, Shri JIT University, Jhunjhunu, Rajasthan, India 
mammograms using Nonsubsampled Contourlet Transform (NSCT) and Support Vector Machine (SVM). The classification of microcalcification is achieved by extracting the microcalcification features from the Contourlet coefficients of the image and the outcomes are used as an input to the SVM for classification.

\section{MATERIALS AND METHODS}

\subsection{Wave Atom Transform}

Wave atom transform is presented by (Demanet and Ying, 2009). The transformation, obeying the parabolic scaling law, can be considered a variant of 2D wavelet packets. Wave atom transform have two very significant properties. First one is the ability to adapt to arbitrary local directions of a pattern. The second one is the ability to sparsely represent anisotropic patterns aligned with the axes. Wave atoms offer sharp frequency localization than other wave packets. It also has significant sparse expansion for oscillatory functions when compared with wavelets, curvelets and Gabor atoms.

The forms of wave packets, known as wavelets, Gabor, ridgelets, curvelets and wave atoms, are created using two parameters, which are $\alpha$ and $\beta$. These variables symbolize decomposition and directional ability for all wave forms. $\alpha$ and $\beta$ values are $1 / 2$ for wave atoms and Fig. 1 shows wave packet's support in space and in frequency plane. Here, $\alpha$ corresponds to the multiscale structure of the transform and $\beta$ corresponds to directional selectivity.

Actually, wave atoms are built from tensor products of $1 \mathrm{D}$ wave packets. One-dimensional wave packets can be represented as $\psi_{\mathrm{m}, \mathrm{n}}^{\mathrm{j}}(\mathrm{X})$, where $\mathrm{j}, \mathrm{m} \geq 0$ and $\mathrm{n} \in \mathrm{Z}$. Frequency restrictions are $\pm \omega_{\mathrm{j}, \mathrm{m}}= \pm \pi 2^{\mathrm{j}} \mathrm{m}$ with $\mathrm{C}_{1} 2^{\mathrm{j}}$ $\leq \mathrm{m} \leq \mathrm{C}_{2} 2^{\mathrm{j}}$. space re-strictions is defined as Equation 1:

$$
X_{j, n}=2^{j}{ }_{n}
$$

Two-dimensional wave atoms $\varphi_{\mu}(\mathrm{X} 1 \mathrm{X} 2)$ are constructed with subscript $\mu=(\mathrm{j}, \mathrm{m}, \mathrm{n})$ where $\mathrm{m}=\left(\mathrm{m}_{1}, \mathrm{~m}_{2}\right)$, $\mathrm{n}=\left(\mathrm{n}_{1}, \mathrm{n}_{2}\right) 2 \mathrm{D}$ orthonormal basis is written as follows Equation 2 and 3:

$$
\begin{aligned}
& \phi_{\mu}^{+}\left(X_{1}, X_{2}\right)=\psi_{m 1}^{\mathrm{j}}\left(X_{1}-2^{-j} n_{1}\right) \psi_{m 2}^{\mathrm{j}}\left(X_{2}-2^{-j} n_{2}\right) \\
& \phi_{\mu}^{-}\left(X_{1}, X_{2}\right)=H \psi_{m 1}^{\mathrm{j}}\left(X_{1}-2^{-j} n_{1}\right) H \psi_{m 2}^{\mathrm{j}}\left(X_{2}-2^{-j} n_{2}\right)
\end{aligned}
$$

where, $\mathrm{H}$ is Hilbert transform. The wave atom tight frame is formed by combination of (2) and (3) Equation 4:

$$
\phi_{\mu}^{(1)}=\frac{\phi_{\mu}^{+}+\phi_{\mu}^{-}}{2}, \phi_{\mu}^{(2)}=\frac{\phi_{\mu}^{+}-\phi_{\mu}^{-}}{2}
$$

\subsection{Support Vector Machine}

Support Vector Machines (SVMs) are a set of related supervised learning methods that analyze data and recognize patterns, used for classification and regression analysis (Rejani and Selvi, 2009). The standard SVM is a non-probabilistic binary linear classifier, i.e., it predicts, for each given input, which of two possible classes the input is a member of. A classification task usually involves with training and testing data which consists of some data instances. Each instance in the training set contains one "target value" (class labels) and several "attributes" (features) (Gorgel et al., 2009). SVM has an extra advantage of automatic model selection in the sense that both the optimal number and locations of the basic functions are automatically obtained during training. The performance of SVM largely depends on the kernel.

SVM is essentially a linear learning machine. For the input training sample set:

$$
\left(\mathrm{x}_{\mathrm{i}}, \mathrm{y}_{\mathrm{i}}\right), \mathrm{i}=1 \ldots \mathrm{n}, \mathrm{x} \in \mathrm{R}^{\mathrm{n}}, \mathrm{y} \in\{-1,+1\}
$$

Let the classification hyper plane equation is to be Equation 5:

$(\omega, x)+b=0$

Thus the classification margin is $2 /|\omega|$. To maximize the margin, that is to minimize $|\omega|$, the optimal hyper plane problem is transformed to quadratic programming problem as follows Equation 6:

$$
\left\{\begin{array}{l}
\min \varphi(\omega)=1 / 2(\omega, \omega) \\
s, t, y_{i}((\omega . x)+b) \geq 1, i=1,2 \ldots 1
\end{array}\right.
$$

After introduction of Lagrange multiplier, the dual problem is given by Equation 7:

$$
\begin{aligned}
& \left\{\max Q(\alpha)=\sum_{i=1}^{n} \alpha_{i}-\frac{1}{2} \sum_{i=1}^{n} \sum_{j=1}^{n} y_{i} y_{j} \alpha_{i} \alpha_{j} K\left(x_{i}, x_{j}\right)\right. \\
& \text { s.t } \sum_{i=1}^{n} y_{i} \alpha_{i}=0, \alpha_{i} \geq 0, i=1,2 \ldots n
\end{aligned}
$$




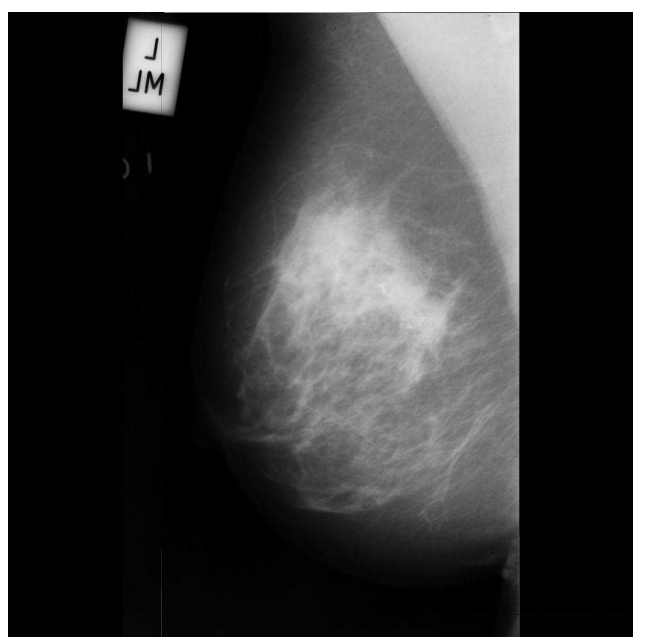

(a)

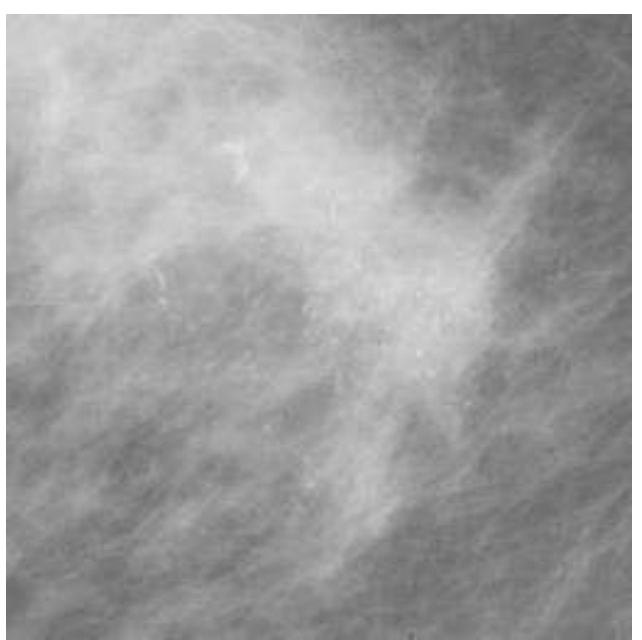

(b)

Fig. 1. (a) Original image (b) extracted ROI

According to Kuhn-Tucker rules, the optimal solution must satisfy:

$$
\alpha_{i}\left(y _ { i } \left(\left(w \cdot x_{i}\right)+b-1=0, i=1,2, . . n\right.\right.
$$

That is to say if the option solution is:

$$
\alpha^{*}=\left(\alpha_{1}^{*}, \alpha_{2}^{*}, \ldots . . \alpha_{i}^{*}\right)^{\mathrm{T}}, i=1,2, \ldots n
$$

Then Equation 8:

$$
w^{*}=\sum_{i=1}^{n} \alpha_{i}^{*} y_{i} x_{i} b^{*}=y_{i}-\sum_{i=1}^{n} y_{i} \alpha_{i}^{*}\left(x_{i}, x_{j}\right), j \in\left\{j\left|\alpha_{i}^{*}\right\rangle 0\right\}
$$

For every training sample point $\mathrm{x}_{\mathrm{i}}$, there is a corresponding multiplier. And the sample points that are corresponding to $\mathrm{a}_{\mathrm{i}}=0$ don't contribute to solve the classification hyper plane while the other points that are corresponding to $a_{i}>0$ do, so it is called support vectors. Hence the optimal hyper plane Equation 9 is given by:

$\sum_{x, \in S V} \alpha_{i} y_{i}\left(x_{i} \cdot x_{j}\right)+b=0$

The hard classifier is then Equation 10:

$$
y=\operatorname{sgn}\left[\sum_{x, \in S V} \alpha_{i} y_{i}\left(x_{i} \cdot x_{j}\right)+b\right]
$$

For nonlinear situation, SVM constructs an optimal separating hyper plane in the high dimensional space by introducing kernel function $\mathrm{K}(\mathrm{x}, \mathrm{y})=\phi(\mathrm{x}) \phi(\mathrm{y})$, hence the nonlinear SVM is given by Equation 11 and 12:

$$
\left\{\begin{array}{l}
\min \varphi(\omega)=1 / 2(\omega, \omega) \\
s, t, y_{\mathrm{i}}\left(\left(\omega . \varphi\left(x_{\mathrm{i}}\right)\right)+b\right) \geq 1, i=1,2 \ldots 1
\end{array}\right.
$$

And its dual problem is given by:

$$
\begin{aligned}
& \max L(\alpha)=\sum_{i=1}^{1} \alpha_{i}-\frac{1}{2} \sum_{i=1}^{1} \sum_{j=1}^{1} y_{i} y_{j} \alpha_{i} \alpha_{j} K\left(x_{i} \cdot x_{j}\right) \\
& \text { s.t. } \sum_{i=1}^{n} y_{i} \alpha_{i}=0,0 \leq \alpha_{i} \leq C, i=1,2, \ldots, 1
\end{aligned}
$$

Thus the optimal hyper plane equation is determined by the solution to the optimal problem.

\section{EXPERIMENTAL SETUP}

The proposed method consists of two stages namely feature extraction stage and classification stage. The two stages are described below:

\subsection{Feature Extraction Stage}

Feature extraction is an essential pre-processing step to pattern recognition and machine learning problems. The main objective of this study is to distinguish between the types of microcalcification as 
benign or malignant. MIAS database, a benchmark database used by many researchers is taken to evaluate this study. The original mammograms in MIAS are very big size $(1024 \times 1024$ pixels $)$. The whole image consists of $50 \%$ of noise.

In order to remove this unwanted noise, a cropping operation is done manually before the feature extraction by choosing the given center of abnormality as the center of the ROI. The size of ROI image is $256 * 256$. In the proposed system, the wave atom coefficients are used as features to classify the images. The original image is decomposed by using the WAT. All coefficients are considered as features for the classification process. This method is applied to all the training images and the feature vectors are stored in the database called as Feature Data Base. The energy of each sub-band of the image $\mathrm{I}$ is calculated by using the formula in (2):

$$
\operatorname{Energy~}_{\mathrm{e}}=\frac{1}{\mathrm{RC}} \sum_{\mathrm{i}=1}^{\mathrm{R}} \sum_{\mathrm{j}=1}^{\mathrm{C}}\left|\mathrm{I}_{\mathrm{e}}(\mathrm{i}, \mathrm{j})\right|
$$

where, $I_{e}(i, j)$ the pixel value of the eth sub-band and $\mathrm{R}, \mathrm{C}$ is width and height of the sub-band respectively. Figure 1 shows the original mammogram image (mdb209) and Fig. 1b shows the cropped image. The block diagram of the proposed method is shown in Fig. 2.

\subsection{Classification Stage}

Classification phase executes two phases. In the first one, the classifier is applied to classify mammograms into normal and abnormal cases. Then the mammogram is considered as abnormal if it contains tumor (microcalcification). Finally, the abnormal mammogram is classified into malignant or benign in the final stage. In this classification stage, SVM classifier in every phase is trained at specific number of training set in each category. Table 1 shows the number of training and testing images.

\section{EXPERIMENTAL RESULTS}

The assessment is carried out in relation with two stages of microcalcification classification system namely (i) normal/abnormal classification (ii) benign/malignant classification.

Table 1. Number of images used to train SVM Classifier

\begin{tabular}{lllll}
\hline Images & Normal & Abnormal & Benign & Malignant \\
\hline Training & 66 & 17 & 8 & 9 \\
Testing & 99 & 25 & 12 & 13
\end{tabular}

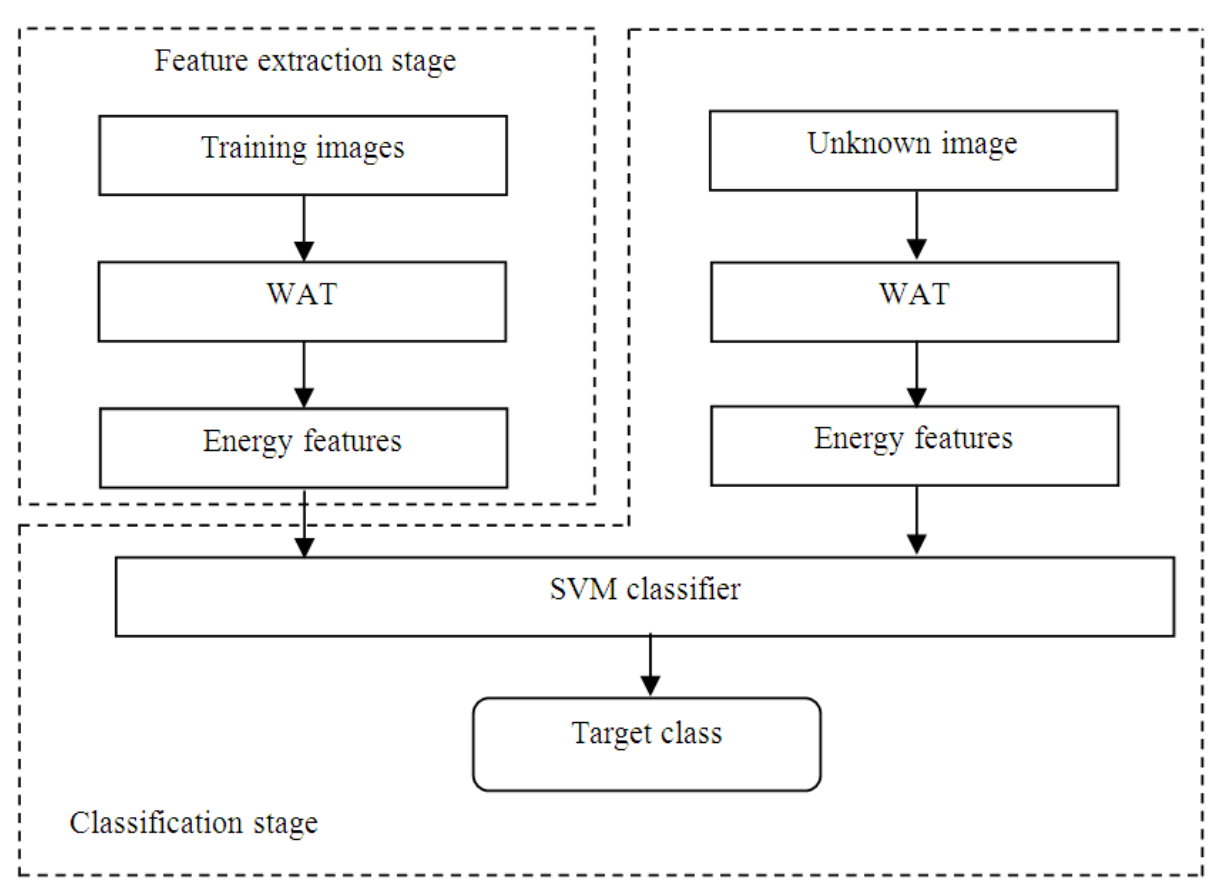

Fig. 2. Block diagram of the proposed method 
Table 2. Success rates of SVM method for the classification of images as normal and abnormal

\begin{tabular}{llll}
\hline \multicolumn{3}{l}{ Wave atom transform+SVM classifier (WAT+SVM) } \\
\hdashline Scale & Accuracy & Sensitivity & Specificity \\
\hline 1 & 100 & 1.00 & 1 \\
2 & 94 & 0.89 & 1 \\
3 & 96 & 0.92 & 1 \\
4 & 88 & 0.81 & 1 \\
\hline
\end{tabular}

Table 3. Success rates of SVM method for the classification of images as benign and malignant

\begin{tabular}{llll}
\hline Wave atom transform+SVM classifier (WAT+SVM) \\
\hdashline Scale & Accuracy & Sensitivity & Specificity \\
\hline 1 & 100 & 1.00 & 1.00 \\
2 & 80 & 0.80 & 0.80 \\
3 & 62 & 0.75 & 0.57 \\
4 & 56 & 0.63 & 0.53 \\
\hline
\end{tabular}

The performance of the proposed system is carried on 99 normal images and 25 microcalcification images. Among the 25 abnormal images, there are 12 benign and 13 malignant images available. All the images are considered for the classification test. Table 2 shows the success rates of SVM method for the classification of images as normal and abnormal. Table 3 shows the success rates of SVM method for the classification of images as normal and abnormal.

At first, the dataset is classified as normal and abnormal. Then the accuracy is calculated for both normal and abnormal images. After that the same process is done for both benign and malignant images.

\section{CONCLUSION}

Breast cancer diagnosis by using digital mammogram is a practical field of investigation of diseases for medical application. This study describes a CAD system for recognizing breast cancer in ROIs of digital mammograms. The study also investigates the performance of the system with wave atom transform and SVM method. The best success rates in this study were obtained with the coefficients at scale of 1,2 and 3 by using SVM. The presented results demonstrate that wave atom transform and SVM are useful and powerful methods to distinguish the mammographic images as normal, benign and malignant.

\section{REFERENCES}

Afrasiabi, K., 2011. Entropyomics as the blueprint of the logic of normal cell division and malignancy. OnLine J. Biol. Sci., 11: 23-26. DOI: 10.3844/ojbsci.2011.23.26

Ballesteros, F., A. Oropesa, M. Lorenzo and D. Andina, 2002. Mammography classification using wavelets. Proceedings of the 5th Biannual World Automation Congress, Jun. 9-13, IEEE Xplore Press, pp: 293300. DOI: 10.1109/WAC.2002.1049559

Demanet, L. and L. Ying, 2009. Wave atoms and time upscaling of wave equations. Numerische Math., 113: 1-71. DOI: 10.1007/s00211-009-0226-6

Faye, I., B.B. Samir, M.M.M. Eltoukhy, 2009. Digital mammograms classification using a wavelet based feature extraction method. Proceedings of the Second International Conference on Computer and Electrical Engineering, Dec. 28-30, IEEE Xplore Press, Dubai, pp: 318-322. DOI: 10.1109/ICCEE.2009.39

Gorgel, P., A. Sertbas, N. Kilic, O.N. Ucan and O. Osman, 2009. Mammographic mass classification using wavelet based support vector machine. J. Elect. Elect. Eng., 9: 867-875.

Hamdi, N., K. Auhmani and M.M. Hassani, 2008. Design of a high-accuracy classifier based on fisher discriminant analysis: Application to computeraided diagnosis of microcalcifications. Proceedings of the International Conference on Computational Sciences and Its Applications, (CSA '08), Washington, pp: 267-273. DOI: 10.1109/ICCSA.2008.43

Jasmine, J.S.L., S. Baskaran and A. Govardhan, 2013. A robust approach to classify microcalcification in digital mammograms using contourlet transform and support vector machine. Am. J. Eng. Applied Sci., 6: 57-68. DOI: 10.3844/ajeassp.2013.57.68

Rejani, Y.A. and S.T. Selvi, 2009. Early detection of breast cancer using SVM classifier technique. Int. J. Comput. Sci. Eng., 1: 127-130.

Zhang, L. and X. Gao, 2007. Research on translationinvariant wavelet transform for classification in mammograms. Proceedings of the International Conference on Natural Computation, Aug. 24-27, IEEE Xplore Press, Haikou, pp: 571-575. DOI: 10.1109/ICNC.2007.631 